Research Article

\title{
Individual expression features of GPX2, NQO1 and SQSTM1 transcript variants induced by hydrogen peroxide treatment in HeLa cells
}

\author{
Anna A. Belanova ${ }^{1}$,Dmitry S. Smirnov ${ }^{1}$, Maxim S. Makarenko ${ }^{2}$, Mariya M. Belousova ${ }^{3}$, \\ Elena V. Mashkina ${ }^{2}$, Anzhela A. Aleksandrova ${ }^{2}$, Alexander V. Soldatov ${ }^{4}$ and Peter V. Zolotukhin ${ }^{1}$ \\ ${ }^{1}$ Evolution Corporate Group, Cell Physiology Laboratory, Southern Federal University, Rostov-on-Don, \\ Russia. \\ ${ }^{2}$ Academy of Biology and Biotechnology, Southern Federal University, Rostov-on-Don, Russia. \\ ${ }^{3}$ Department of English Language for Natural Sciences Faculties, Southern Federal University, \\ Rostov-on-Don, Russia. \\ ${ }^{4}$ Department of Nanosystems Physics and Spectroscopy, Southern Federal University, Rostov-on-Don, \\ Russia.
}

\begin{abstract}
Pathway activity assessment-based approaches are becoming highly influential in various fields of biology and medicine. However, these approaches mostly rely on analysis of mRNA expression, and total mRNA from a given locus is measured in the majority of cases. Notably, a significant portion of protein-coding genes produces more than one transcript. This biological fact is responsible for significant noise when changes in total mRNA transcription of a single gene are analyzed. The NFE2L2/AP-1 pathway is an attractive target for biomedical applications. To date, there is a lack of data regarding the agreement in expression of even classical target genes of this pathway. In the present paper we analyzed whether transcript variants of GPX2, NQO1 and SQSTM1 were characterized by individual features of expression when HeLa cells were exposed to pro-oxidative stimulation with hydrogen peroxide. We found that all the transcripts (10 in total) appeared to be significantly individually regulated under the conditions tested. We conclude that individual transcripts, rather than total mRNA, are best markers of pathway activation. We also discuss here some biological roles of individual transcript regulation.
\end{abstract}

Keywords: NFE2L2/AP-1 pathway, interactomics, transcript variants expression control.

Received: January 19, 2016; Accepted: November 22, 2016.

\section{Introduction}

Pathway activity assessment-based approaches are becoming more and more influential in various and diverse fields of biology and medicine: in environmental monitoring (Shukla et al., 2012), general (Subramaniam and Ellis, 2011; Chakraborty et al., 2014; Shkurat et al., 2014) and personalized (Wu et al., 2010) pharmacology, pathophysiology (Zolotukhin et al., 2014a), diagnostics (Zolotukhin et al., 2014b; Yao et al., 2015), and patient follow-up (Sibhatu et al., 2008; Shimizu et al., 2015). Most of these approaches rely on RNA expression analysis, because measuring transcription rate is the most representative means to assess pathway activation known to date. However, individual control of the transcript variant expression is ignored in most cases.

Send correspondence to Peter V. Zolotukhin. Evolution Corporate Group, Cell Physiology Laboratory, Southern Federal University, 194/1 Stachki av., 344090 Rostov-on-Don, Russia. E-mail: p.zolotukhin@gmail.com.
Yet, a pathway may control transcripts' fate individually on several levels. Firstly, transcription factors of the pathway can directly induce individual transcripts (Jyrkkänen et al., 2011). Secondly, transcription factors, being central to some pathways, can attract and regulate splicing machinery themselves (Pan et al., 2003). Thirdly, other pathway components can easily regulate splicing machinery together with promoting target gene transcription (Yadav et al., 2014). Fourthly, cellular pathways have all capabilities to individually control degradation or long-term storage of mature mRNA variants of a single gene (Shim and Karin, 2002; Rattenbacher and Bohjanen, 2012; Kurinna and Werner, 2015).

Thus, individual transcripts, rather than total mRNA read from a single locus appear to be far more valuable and adequate for purposes of pathway activity assessment. Yet, individual transcript expression-based studies are rare due to technical and interpretative difficulties (Arseneau et al., 2009; Boudreau et al., 2011). 
One of highly informative pathways used today in all the above mentioned biology and medicine fields is the NFE2L2/AP-1 pathway (sometimes subdivided into NFE2L2 (Nrf2) and AP-1 pathways in the literature). Despite attracting much attention, this pathway is poorly studied in terms of regulation of expression of transcripts of target genes of this pathway. Our laboratory previously contributed to solving the problem by showing that TXN transcript variants are differentially regulated upon NFE2L2/AP-1 activation (Dovzhik et al., 2014). Still, not much is done for other genes, even those frequently used as pathway activation markers in numerous studies (Marrot et al., 2008; MacLeod et al., 2009; Zhang et al., 2010; Subramaniam and Ellis, 2011; Wang et al., 2014). At the same time, pathway activation would be much easier to detect and, which is even more important, with greater specificity, if individual transcripts were used as pathway activation markers. The basis for this consideration is the fact that when, for example, one of the three transcripts of a given locus may be induced, the second one suppressed and the third one unchanged, total mRNA from this locus suggests expression to be unchanged and the pathway to be not activated at all, which is obviously a false negative result.

In addition to the practical implication, differential regulation of transcript variants is of great biological significance. Transcript variants fold differently and thus interact differently with RNA-transporting and RNA-processing proteins. This difference in interactions makes it possible for the cell to tightly regulate the speed of response towards stimuli: transcript variants differ quantitatively and qualitatively in storage/retention properties, rate of degradation and speed of translation. This biological matter is also rarely addressed in studies.

Considering these issues, we decided to test whether three important NFE2L2/AP-1 pathway targets with with more than one mRNA form could indicate individual transcript regulation. These genes were GPX2, NQO1 and SQSTM1.

GPX2, a cytosolic enzyme reducing peroxides using glutathione as the substrate, is one of seven known proteins of the human family of glutathione peroxidases (GPX8 is still considered a probable glutathione peroxidase). The gene coding for this protein is peculiar: its basal and inducible expression is mostly and almost directly regulated by NFE2L2 (Singh et al., 2006; Sykiotis and Bohmann, 2010), and its ARE is well-studied (Singh et al., 2006). In contrast, the NQO1 gene, one of the first known NFE2L2 targets, has multiple transcription factors controlling it, although in some cells, NQO1 is also controlled mostly by NFE2L2 (Marrot et al., 2008). The NQO1 NFE2L2 binding site is also thoroughly described: it is characterized by two core ARE sequences with one embedded TRE sequence (Venugopal and Jaiswal, 1996; Kim et al., 2011). NQO1 codes for a multi-functional enzyme scavenging superoxide anion (Siegel et al., 2004; Dinkova-Kostova and Talalay, 2010), reducing quinones and thus blocking redox-cycling (Jaiswal, 2000), and protecting the nucleus from pro-oxidants (Winski et al., 2002) as well as associating with mitotic spindle (Siegel et al., 2012). NQO1 is even known to stabilize p53 (officially known as TP53) (Dinkova-Kostova and Talalay, 2010). The autophagosomal adaptor protein SQSTM1 (also known as p62) is capable of activating the NFE2L2 sub-pathway without oxidative modification of the KEAP1 protein (Copple et al., 2010; Bui and Shin, 2011). The mode of antioxidant action of this factor is in line with its primary function, as it merely targets KEAP1 for autophagosomal degradation (Copple et al., 2010; Bui and Shin, 2011). Interestingly, in a murine model, Sqstm1dependent activation of Nrf2 was responsible for approximately $50 \%$ of basal expression of classical Nfe212 targets: Nqo1, Gclc, and Hmoxl (Copple et al., 2010). The ARE of SQSTM1 was proven to be functional, and this gene is another NFE2L2 target (Jain et al., 2010).

The aim of the study was to assess whether GPX2, NQO1 and SQSTM1 transcript variants were regulated individually when cells were exposed to hydrogen peroxide treatment, a classical NFE2L2/AP-1 activation stimulus.

\section{Materials and Methods}

The study was carried out in 2015 at the Southern Federal University Academy of Biology and Biotechnology Shared Equipment Centre.

\section{Cell culture, hydrogen peroxide treatment and viability assay}

In this study, the HeLa cell line was used as an experimental model. The cells were kindly provided by Southern Scientific Center of the Russian Academy of Science and validated by cytogenetic (G-staining) and molecular genetic analyses. The cells were grown in T25 flasks, 24- and 96-well plates (SPL Lifesciences, South Korea) in GlutaMax DMEM medium (Thermo Fisher Scientific, USA) supplemented with $10 \%$ of fetal bovine serum (GE Healthcare, UK) and $0.05 \mu \mathrm{g} / \mathrm{ml}$ of gentamicin (Biokhimik JSC, Russia). The cells were kept at $37{ }^{\circ} \mathrm{C}$ and $5 \% \mathrm{CO}_{2}$, with passive humidification in the Sanyo MCO-18AC incubator (Panasonic, Japan). Cell growth was controlled using the Premiere MIS-9000 inverted microscope (C\&A, China).

As the NFE2L2/AP-1 pathway is activated by prooxidants, we used hydrogen peroxide as a convenient treatment substance. It is a physiological compound and its injection into the medium does not introduce any additional metabolites that could affect the performance of the classical variant of the pathway by activating various upstream kinases and adjacent pathways further indirectly affecting the NFE2L2/AP-1 pathway. Hydrogen peroxide stock solution (ProChem LLC, Russia) concentration was assessed immediately prior to each injection using the spectrophoto- 
metric assay at $240 \mathrm{~nm}$ on SmartSpec instrument (Bio-Rad, USA). Hydrogen peroxide (100 uM) is stable for long time periods in a standard culturing medium without cells (Erol et al., 2012), thus relatively fast hydrogen peroxide depletion in the cell culture represents an adequate stimulus for NFE2L2/AP-1 pathway activation. In our previous study we found that HeLa cells retain normal viability while having the NFE2L2/AP-1 pathway activated at hydrogen peroxide concentration in the medium of $400 \mathrm{uM}$ when treated for $24 \mathrm{~h}$ (Belanova et al., 2017). A 24-h incubation period was used, based on the observation that full-scale activation of several NFE2L2/AP-1 pathway targets is achieved and stabilized by $24 \mathrm{~h}$, and this treatment period is used in numerous related studies (Marrot et al., 2008). For routine cell viability screening, we used a trypan blue exclusion assay. There were 8 samples in each group.

\section{RNA isolation}

The RNA isolation procedures and cDNA synthesis set-up were performed in a laminar flow cabinet decontaminated with RNaseZap anti-RNase reagent (Sigma, USA). RNA was isolated using the Qiazol lysis reagent (Qiagen, The Netherlands) according to the standard phenol-lysis modification of the acidic phenolic method (Chomczynski and Sacchi, 2006). Phase separation was achieved by adding of 1-bromo-3-chloropropane (Sigma, USA). RNA was precipitated with isopropanol (Vekton CJSC, Russia) and then twice washed with $75 \%$ purified ethanol. The RNA pellet was dissolved in DEPC-treated water (Syntol LLC, Russia), heated, mixed and aliquoted for checking of RNA integrity and purity, genomic DNA contamination control, and reverse transcription.

RNA integrity was assessed using non-denaturing $1 \%$ agarose gel electrophoresis (Amresco, USA; Lytech LLC, Russia; Helikon LLC, Russia; DNA-technology LLC, Russia). The gel was stained with ethidium bromide (Lytech LLC, Russia) and bands visualized on a GelDoc XR system (Bio-Rad, USA). All samples had bright distinct rRNA bands, a mixed 5.8S/5S/tRNA band, a normal mRNA smear, and no visual signs of degradation (Supplementary Figure S1). The spectrophotometric assays were performed using the Nanodrop-1000 instrument (Thermo Fisher Scientific, USA). All samples had a A260/280 ratio $\geq 1.8$, ranging between 1.83 and 1.97 , as well as no signs of significant ethanol carry-over.

Genomic DNA or other template/primer contamination was checked as a standard reverse transcription reaction mixture without the enzyme run in qPCR reactions with the primers to the chosen regions of the mRNAs. The details on the qPCR protocol are provided below. No samples had a qPCR curve reaching the quantification threshold before the $40^{\text {th }}$ cycle.

RNA was reverse-transcribed using a kit from Syntol LLC (Russia) according to the manufacturer's protocol with an oligo(dT) primer. The reaction was run for $1 \mathrm{~h}$ at
$39{ }^{\circ} \mathrm{C}$, and then the enzyme was inactivated by a $5 \mathrm{~min}$ incubation at $92{ }^{\circ} \mathrm{C}$. The cDNA was stored at $-20^{\circ} \mathrm{C}$.

\section{Primer design and synthesis, and quantitative PCR}

We designed primers for $G P X 2$ (one protein-coding transcript and two NMD-transcripts), NQO1 (four transcript variants), SQSTM1 (three transcript variants), TBP (reference gene) and POLR2C (reference gene) according to the standard selection procedure using the most recent GenBank reference RNA sequences (NCBI Gene), Oligo 7 software for primer selection, OligoCalc and IDT Oligo Analyzer for the melting temperature analysis consistency test, and NCBI primer BLAST for the in silico specificity test (against the refseqRNA database). The primers were selected so as to span exon-exon junctions. The GPX2 NMD (nonsense-mediated decay) transcript variant 2 primers required a single LNA modification to normalize the thermodynamic properties of the pair. The primer sequences are given in Supplementary Table S1. Oligonucleotides were synthesized by Syntol LLC (Russia) and dissolved in DEPC-treated water (Syntol, Russia).

Quantitative PCR (qPCR; the SYBRGreen type; FAM channel detection) was performed using hot-start EvaGreen qPCR kits from Syntrol LLC (Russia) or OneTaq Hot Start DNA Polymerase with GC-buffer (New England Biolabs, USA) on a CFX96 instrument (Bio-Rad, USA). The latter was only used for the SQSTM1 transcript variant 1 (tv1) cDNA, as this amplicon required higher annealing temperatures than the other targets. In this case, the SQSTM1 tv1 reaction mixes were always run with both $P O L R 2 C$ and $T B P$, with a higher melting temperature set for the SQSTM1 transcript variant 1 wells using the gradient function. In these settings, POLR2C and TBP had $0.3{ }^{\circ} \mathrm{C}$ difference in the annealing temperatures, which was negligible as it was seen from the PCR optimization set-ups (data not shown). The reaction parameters were as follows: $94{ }^{\circ} \mathrm{C}$ for $5 \mathrm{~min}$ (the polymerase activation step); 35 cycles ( 40 cycles in the negative control reaction set-ups) of $94{ }^{\circ} \mathrm{C}$ for $15 \mathrm{~s}, 57.5^{\circ} \mathrm{C}\left(60^{\circ} \mathrm{C}\right.$ for SQSTM1 tv1 and thermal gradient in the amplification specificity check set-ups) for $20 \mathrm{~s}$, $70{ }^{\circ} \mathrm{C}$ for $30 \mathrm{~s}$, followed by a melting analysis $\left(0.5^{\circ} \mathrm{C}\right.$ increment from 50 to $95^{\circ} \mathrm{C} ; 20 \mathrm{~s}$ per cycle).

The reaction optimization and characterization included gradient PCR for determining the effective annealing temperature and a primer efficiency test performed in 4-step two-fold serial dilutions for each primer pair. All reactions had efficiency within an acceptable range. Reaction specificity was controlled using the melting curve analysis and $1.5 \%$ agarose gel electrophoresis for each primer pair in several repeats. No abnormal products were detected.

\section{RNA folding analysis}

RNA folding predictions were performed using the RNAfold web tool (Vienna RNA servers). MFE structures are presented in Results. 


\section{Data analysis}

qPCR data were analyzed using CFX96 system software (Bio-Rad, USA). Quantification threshold was set at the level of the early logarithmic phase of the qPCR curves, and it was the same in all reactions.

The $\mathrm{Ct}$ data were normalized using the standard deltaCt algorithm expressed by the formula $\mathrm{R}=(2 * \mathrm{E})^{\wedge}($-deltaCt), where $\mathrm{R}$ is the ratio of expression of the target and reference (or regulating - NFE2L2) genes; E is the target gene reaction efficiency expressed as a proportion; deltaCt is the arithmetic diff between the target and reference genes. Data for the target genes were normalized to the both reference genes independently and to their geometric mean.

Statistical analysis was performed using SPSS22 software (IBM, USA). All data were tested for normality (Kolmogorov-Smirnov test). Parametric (ANOVA, Pearson's correlation) and non-parametric (MannWhitney, Spearman's correlation, and the Fisher r-to-z transformation) tests were employed in accordance with the normality testing results. All data were normally distributed and thus tested with both parametric and non-parametric criteria so as to assess the statistical consistency. All calculations were performed with appropriate adjustments for small groups in order to reduce a false-positive hypothesis rejection rate. Expression data (in relative units, r.u.) are given as mean $(\mathrm{m}) \pm \mathrm{SD}$. First-type error was considered acceptable when below 0.05 .

\section{Results}

\section{Properties testing of reference genes}

Reference gene selection is a major challenge in gene expression studies. In the present study we used two reference genes, TBP and POLR2C, that had been previously proven in our lab to be adequate for experimental model of hydrogen peroxide treatment of HeLa cells (Belanova et $a l ., 2017)$. In the present study, we re-evaluated these data. The results are given in Table 1. As seen from the data, TBP and POLR2C have highly congruent expression in the control and treatment groups.

\section{GPX2, NQO1 and SQSTM1 transcript variants folding}

Predicted folding patterns of the GPX2, NQO1 and SQSTM1 transcript variants are shown in Figure 1, Figure 2 and Figure 3, respectively. As seen from the figures, transcript variants of the three genes have significantly different folding.

\section{Expression of the GPX2, NQO1 and SQSTM1 transcript variants in hydrogen peroxide-treated HeLa cells}

The expression analysis results are given in Table 2, and representative graphs are provided in Figure 4. As seen from Table 2, in the settings tested, the GPX2 NMDtranscripts had no detectable expression. At the same time, GPX2 transcript variant 1 was pronouncedly induced by $400 \mathrm{uM}$ hydrogen peroxide treatment (Figure 4A). The results were consistent when statistically tested using both parametric and non-parametric methods, and were similar disregarding the normalization method.

SQSTM1 transcript variants 1 and 2 (Figure 4B and $4 \mathrm{C}$, respectively) did not show any changes in expression upon $400 \mathrm{uM}$ hydrogen peroxide exposure. Transcript variant 3, however, was completely undetectable.

For NQO1 (Figure 4D), we found clear evidence of differential regulation of expression of transcript variants. NQO1 transcript variant 1 showed some decrease in expression in the treatment group, although only close to significance and not in the case of TBP-normalization. Transcript variant 2 did not show any significant expression differences between the groups. Transcript variant 3 demonstrated decreased expression in the hydrogen peroxide treatment group when normalized to POLR2C. In contrast, transcript variant 4 had higher expression in the treatment group with agreement of the TBP and double normalization methods.

\section{Discussion}

Analyzing individual rather than total transcripts arising from a given gene is a far more effective approach when pathway signaling activity is considered. The pathway under analysis may control only one transcript or a set of transcripts of its target-gene, and the controlled transcripts may be regulated in opposite directions. Moreover, even when total resulting protein-coding capacity of an activated gene is in the focus, individual transcripts are still much more reliable than total RNA. The reason is that individually controlled transcripts also differ in their fate (storage, degradation or translation) and, in case of storage and translation, storage period and translation speed, respectively. Further-

Table 1 - Results of correlation analysis of $T B P$ and $P O L R 2 C$ expression.

\begin{tabular}{|c|c|c|c|c|c|}
\hline \multirow[t]{2}{*}{ Correlation analysis type } & \multicolumn{2}{|c|}{ Control } & \multicolumn{2}{|c|}{ Hydrogen peroxide, $400 \mathrm{uM}, 24 \mathrm{~h}$} & \multirow{2}{*}{$\begin{array}{l}\text { Differences between } \\
\text { the groups }\end{array}$} \\
\hline & Correlation coefficient & p-level & Correlation coefficient & p-level & \\
\hline Parametric testing & 0.857 & 0.029 & 0.843 & 0.009 & Non-significant \\
\hline Non-parametric testing & 0.886 & 0.019 & 0.929 & 0.001 & Non-significant \\
\hline
\end{tabular}


A

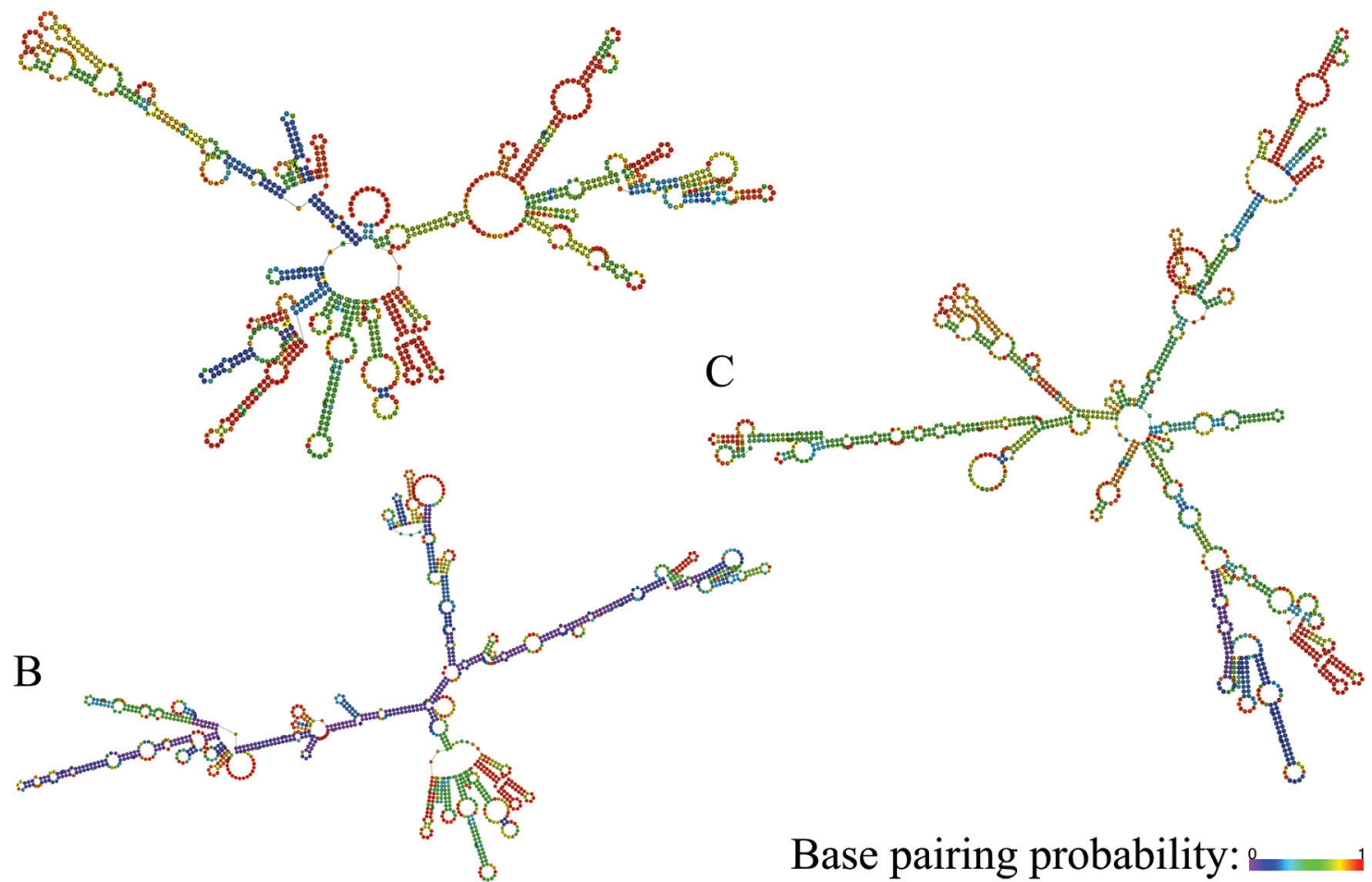

Figure 1 - Folding patterns of GPX2 transcript variants. A - transcript variant 1 (protein-coding); B - transcript variant 2 (NMD); C - transcript variant 3 (NMD). Color-scale represents base pairing probabilities: violet and red correspond to 0 and 1 probabilities, respectively.

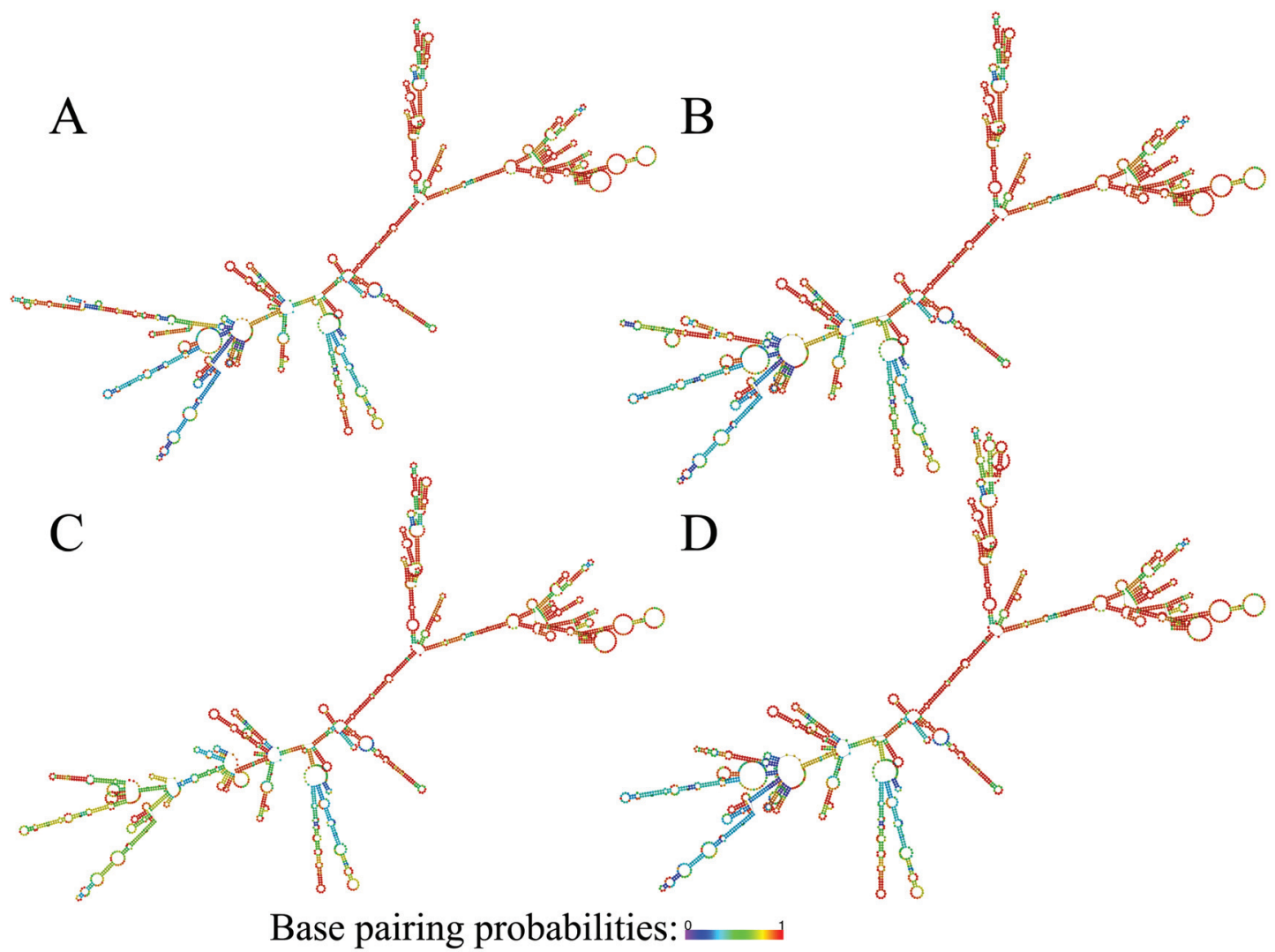

Figure 2 - Folding patterns of $N Q O 1$ transcript variants. A - transcript variant 1; B - transcript variant 2; C - transcript variant 3; D - transcript variant 4. Color-scale represents base pairing probabilities: violet and red correspond to 0 and 1 probabilities, respectively. 

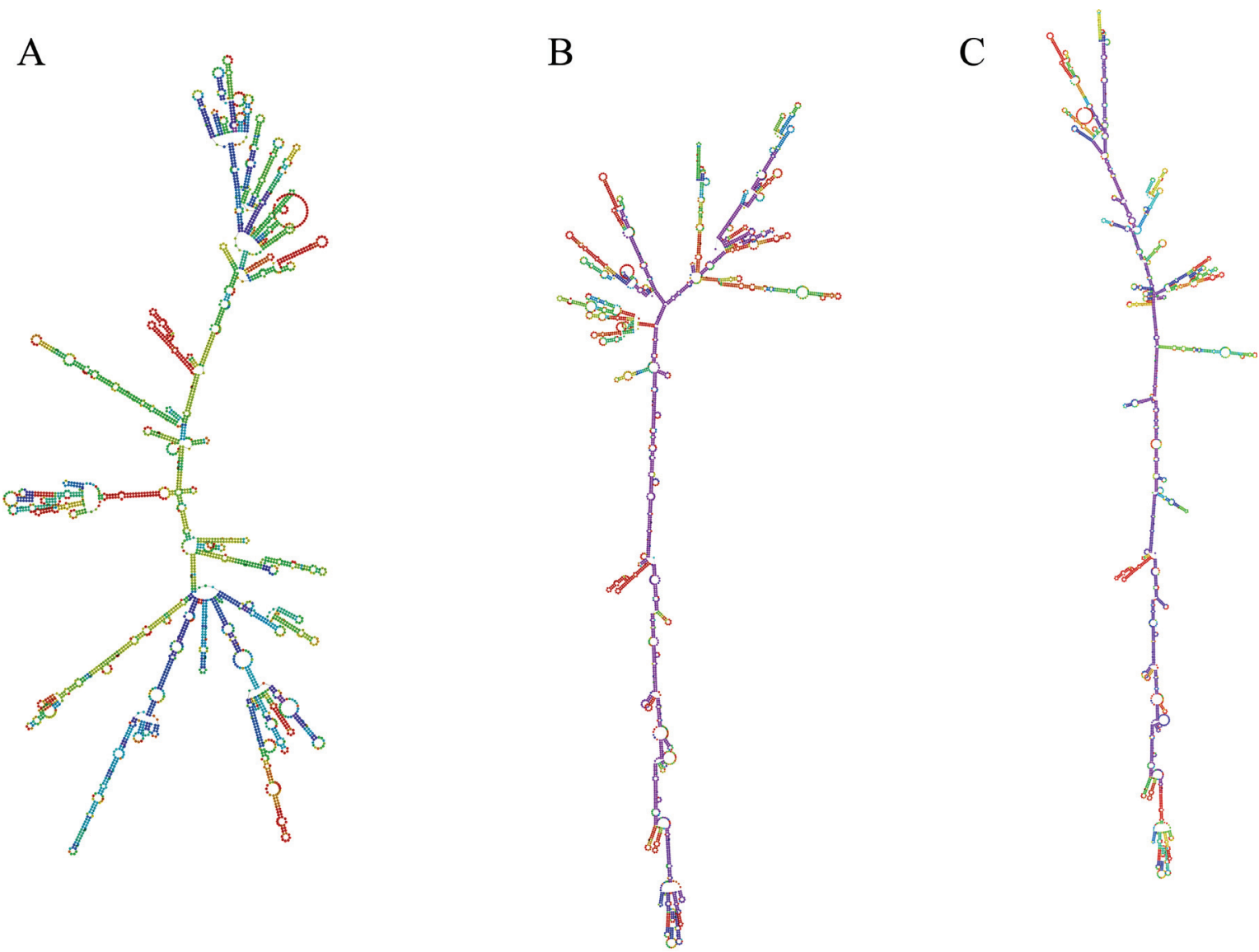

\section{Base pairing probabilities:}

Figure 3 - Folding patterns of SQSTM1 transcript variants. A - transcript variant 1; B - transcript variant 2; C - transcript variant 3. Color-scale represents base pairing probabilities: violet and red correspond to 0 and 1 probabilities, respectively.

more, biological significance of differential regulation of transcripts may be even greater, being linked to disease (Trombetta-Lima et al., 2015) or miRNA or other processing control mechanisms (Laloo et al., 2010). Due to these challenges in current cell biology, we aimed to test whether the NFE2L2/AP-1 pathway target genes GPX2, NQO1 and SQSTM1 exhibited individual transcript control when the cells were exposed to sub-lethal hydrogen peroxide treatment.

The transcripts of all these genes arise due to alternative splicing. Two of three transcripts of GPX2 contain alternate internal exons rendering them subject to nonsensemediated RNA decay (NCBI Gene). Three of four NQO1 transcripts lack one or two in-frame exons, but still, all four RNAs are protein-coding (NCBI Gene). Two SQSTM1 transcripts differ from the predominant transcript in 5'-UTR structure, yet, these transcripts also arise from alternative splicing (NCBI Gene). Although differential initiation of transcription has already been described for the NFE2L2/AP-1 pathway target gene BACH1 (Jyrkkänen et $a l ., 2011)$, and this mechanism of transcript variants formation under control of a transcription factor is the one ex- pected in the first place, alternative splicing is also often regulated by transcription factors. For example, an adjacent to the NFE2L2/AP-1 pathway, the SP1 pathway, controls a splicing factor SLU7, which, in turn, controls alternative splicing pattern(s) of the cell (Alberstein et al., 2007). An example of a more specific splicing control is seen in the HIF1A pathway: HIF1A itself controls alternative splicing of its targets, including the well-known PDK1 gene (Sena et al., 2014). There are other examples of situations where transcription factors directly or indirectly control alternative splicing of their own targets and of other genes (Liu et al., 2013). As known from these cases, transcription factors do so by regulating expression of splicing factors, or by direct effects on their target RNAs, or even by employing epigenetic machinery (Pan et al., 2003; Luco et al., 2010). Interestingly, the third case was actually described for an NFE2L2/AP-1 pathway component, the JDP2 transcription factor (Lerdrup et al., 2005). JDP2 suppresses JUN activity and permits NFE2L2 activity (Tanigawa et al., 2013), and also modulates alternative splicing of its targets via epigenetic mechanisms (Pan et al., 2003; Luco et al., 2010). Additionally, some genes do not have canonical TATA or 
Table 2 - Expression of the GPX2, NQO1, SQSTM1 transcript variants in control cells and in HeLa cells treated with 400 uM of hydrogen peroxide.

\begin{tabular}{|c|c|c|c|c|c|c|}
\hline \multirow{2}{*}{$\begin{array}{l}\text { Group } \\
\text { Normalization: }\end{array}$} & \multicolumn{3}{|c|}{ Control } & \multicolumn{3}{|c|}{ Hydrogen peroxide, 400 , uM $24 \mathrm{~h}$} \\
\hline & $T B P$ & POLR2C & Double normalization & $T B P$ & POLR2C & Double normalization \\
\hline$G P X 2$ tv 1 & $0.007 \pm 0.004$ & $0.01 \pm 0.004$ & $0.01 \pm 0.004$ & $0.13 \pm 0.04$ & $0.16 \pm 0.06$ & $0.15 \pm 0.05$ \\
\hline \multirow[t]{2}{*}{ p-level } & - & & & 1: 0.019 & 1: 0.04 & 1: 0.028 \\
\hline & & & & 2: 0.001 & 2: 0.005 & 2: 0.005 \\
\hline$G P X 2$ tv 2 & \multicolumn{3}{|c|}{ Expression undetectable } & \multicolumn{3}{|c|}{ Expression undetectable } \\
\hline p-level & - & & & & & \\
\hline$G P X 2$ tv 3 & \multicolumn{3}{|c|}{ Expression undetectable } & \multicolumn{3}{|c|}{ Expression undetectable } \\
\hline p-level & - & & & & & \\
\hline$N Q O 1$ tv1 & $3.2 \pm 0.99$ & $5.7 \pm 1.97$ & $4.2 \pm 1.38$ & $1.8 \pm 0.12$ & $2.1 \pm 0.26$ & $1.9 \pm 0.15$ \\
\hline \multirow[t]{2}{*}{ p-level } & & - & & 1: 0.14 & 1: 0.054 & 1: 0.082 \\
\hline & & & 2: 0.28 & 2: 0.08 & 2: 0.081 & \\
\hline$N Q O 1$ tv2 & $1.3 \pm 0.45$ & $2.2 \pm 0.8$ & $1.7 \pm 0.6$ & $1.03 \pm 0.08$ & $1.15 \pm 0.1$ & $1.1 \pm 0.06$ \\
\hline \multirow[t]{2}{*}{ p-level } & & - & & 1: 0.47 & 1: 0.17 & 1: 0.27 \\
\hline & & & & 2: 1.0 & 2: 0.23 & 2: 0.85 \\
\hline NQO1 tv3 & $9.7 \pm 1.8$ & $17.7 \pm 3.7$ & $12.9 \pm 2.5$ & $8.9 \pm 0.5$ & $10.2 \pm 1.16$ & $9.4 \pm 0.65$ \\
\hline \multirow[t]{2}{*}{ p-level } & - & & & 1: 0.63 & $1: 0.048$ & 1: 0.15 \\
\hline & & & & 2: 0.75 & 2: 0.06 & 2: 0.34 \\
\hline NQO1 tv4 & 1.50 .33 & $2.6 \pm 0.39$ & $1.9 \pm 0.33$ & 2.80 .2 & $3.1 \pm 0.3$ & $2.9 \pm 0.2$ \\
\hline \multirow[t]{2}{*}{ p-level } & - & & & 1: 0.06 & 1: 0.305 & 1: 0.019 \\
\hline & & & & 2: 0.02 & 2: 0.181 & 2: 0.043 \\
\hline SQSTM1 tv1 & $7.3 \pm 2.9$ & $11.4 \pm 5.1$ & $8.9 \pm 3.7$ & $4.1 \pm 1.0$ & $4.2 \pm 0,8$ & $4.1 \pm 0,9$ \\
\hline \multirow[t]{2}{*}{ p-level } & - & & & $1: 0.27$ & 1: 0.13 & 1: 0.18 \\
\hline & & & & $2: 1.0$ & 2: 0.18 & 2: 0.66 \\
\hline SQSTM1 tv2 & $0.2 \pm 0.05$ & $0.3 \pm 0.06$ & $0.2 \pm 0.05$ & $0.2 \pm 0.02$ & $0.2 \pm 0.01$ & $0.18 \pm 0.01$ \\
\hline \multirow[t]{2}{*}{ p-level } & - & & & 1: 0.9 & 1: 0.13 & 1: 0.43 \\
\hline & & & & 2: 1.0 & 2: 0.41 & $2: 1.0$ \\
\hline SQSTM1 tv3 & \multirow{2}{*}{\multicolumn{3}{|c|}{ Expression undetectable }} & \multirow{2}{*}{\multicolumn{3}{|c|}{ Expression undetectable }} \\
\hline p-level & & & & & & \\
\hline
\end{tabular}

${ }^{1}$ ANOVA testing $\mathrm{p}$-level; ${ }^{2}$ Mann-Whitney criterion testing p-level

CAAT boxes, and alternative genomic elements may serve for transcription initiation, bringing transcription factors and splicing control even closer (Malakooti et al., 2001).

We found that, having a 15 -fold increase in expression, GPX2 was the most easily induced gene among those tested in our laboratory in this and our previous study [previously, we worked with $H M O X 1, F T H 1, C B R 3, S E S N 2$, $G C L C, J U N$ and NFE2L2 using the same experimental model (Belanova et al., 2017)]. In the present experimental settings, we could not detect expression of the NMD transcripts. However, under other conditions, the two $N M D$ transcripts can be significantly up-regulated, and this expression character may not be similar to that of the protein-coding transcript.

$N Q O 1$ was confirmed to have differential regulation of the transcripts in the settings tested. Transcripts 1 and 3 expression decreased (closely to significance in case of the transcript 1), while transcript 4 expression significantly increased. This is exactly the situation when total mRNA expression analysis would be insensitive to apparent, real changes in gene expression. Thus, all four transcripts differ in regulation, and one should establish the most responsive transcript for a study to be undertaken. One interesting question raised in the present study in this sense is what exactly are the transcripts controlled by NFE2L2 and AP-1. These two transcription factors may also control entirely or partially different transcripts.

SQSTM1 also had a complex expression pattern. Transcript variant 3 was undetectable in the present settings. However, this transcript codes for a protein, and thus, its highly individual character of expression should be considered in further experiments. Transcript variants 1 and 2 did not demonstrate any differences in expression. However, SQSTM1 was previously shown to be JUNsuppressed in the settings tested. Thus, the two transcripts may actually have pronounced differences in expression. We plan to test whether only one of the detected transcripts is negatively regulated by JUN. Nevertheless, it is obvious that analyzing individual, rather than total, transcripts is a 

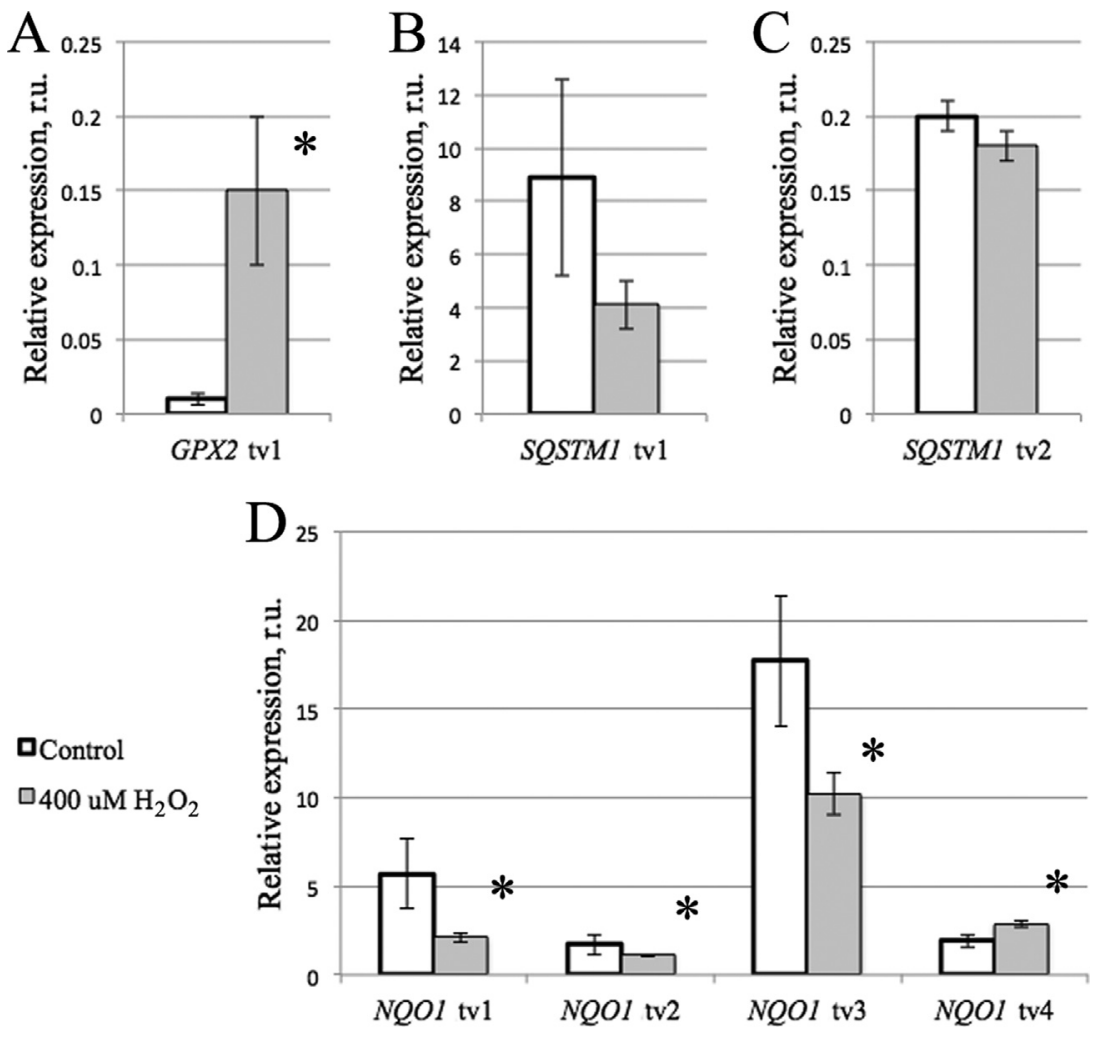

Figure 4 - Character of expression of $\operatorname{GPX2}(\mathrm{A}), \operatorname{SQSTM1}(\mathrm{B}, \mathrm{C})$ and $N Q O 1$ (D) transcript variants. * $-\mathrm{p}<0.05$ - differences are significant in at least one of the normalization methods.

preferred strategy for pathway activation studies, as well as for protein expression-based cellular responses tests.

From a structural point of view, all transcripts of all three studied genes have quite pronounced differences in folding. This highly plausibly implies differences in features of interactions with proteins determining RNA shuttling, storage, degradation and translation (Lin and Bundschuh, 2013; Ozretić et al., 2015), rendering a given gene to serve different roles under different conditions and cellular contexts. In this sense, our results stress the need for further studies that will uncover functional insights into biological roles of differential expression of the GPX2, NQO1 and SQSTM1 transcript variants.

There were several limitations of our study that we would like to outline and discuss. Firstly, as this was the first study for the set of genes we chose and one of only few studies on differential regulation of transcripts expression in general, we used only one fundamental model of activation of the NFE2L2/AP-1 pathway. We plan to study other stimuli in the future and anticipate that there will be slight differences in responses of the transcripts due to changes in the general cellular context. Secondly, we did not study the biological roles of the differential expression of transcript variants. As for the current study, we did not aim or plan to do it, since we could not predict the results of the study. This major and extremely complex problem, which con- cerns the biological significance of individual transcript variant control, requires a separate thorough investigation. However, we feel that these limitations do not compromise our findings, which will definitely be helpful for future studies in molecular biology of the cell and molecular medicine.

To summarize the results of the study, all three genes tested, GPX2, NQO1 and SQSTM1, were characterized by individual control of transcript variants expression in $\mathrm{HeLa}$ cells treated with $400 \mathrm{uM}$ hydrogen peroxide. These features of the genes should be accounted for in experiments designed for the NFE2L2/AP-1 pathway activation-based studies, as well as in related and similar projects, as some transcripts of these genes have opposite regulation. These features of the studied genes, along with highly distinct folding patterns of their transcripts, also suggest significant differences in the biological roles of the transcript variants.

\section{Acknowledgments}

This study was financially supported by the Russian Science Foundation (Grant \# 14-35-00051, "Development of optimized synthesis techniques of metal-containing nanoparticles having a pronounced effect on tumor tissue, and integrated technologies for enhancing antitumor resistance mechanisms using nanomaterials and personalized low-intensity systemic effects"). 


\section{References}

Alberstein M, Amit M, Vaknin K, O’Donnell A, Farhy C, Lerenthal Y, Shomron N, Shaham O, Sharrocks AD, AsheryPadan R, et al. (2007) Regulation of transcription of the RNA splicing factor hSlu7 by Elk-1 and Sp1 affects alternative splicing. RNA 13:1988-1999.

Arseneau JR, Laflamme M, Lewis SM, Maïcas E and Ouellette RJ (2009) Multiple isoforms of PAX5 are expressed in both lymphomas and normal B-cells. Br J Haematol 147:328338.

Belanova AA, Smirnov DS, Makarenko MS, Kornienko IV, Kurbatov SV, Belousova MM, Aleksandrova AA, Kharchenko EY, Prazdnova EV and Zolotukhin PV (2017) JUN knockdown reinforces expression of NFE2L2-dependent genes in hydrogen peroxide-treated HeLa cells. Indian J Exp Biol, in press.

Boudreau LH, Bertin J, Robichaud PP, Laflamme M, Ouellette RJ, Flamand N and Surette ME (2011) Novel 5-lipoxygenase isoforms affect the biosynthesis of 5-lipoxygenase products. FASEB J 25:1097-1105.

Bui CB and Shin J (2011) Persistent expression of Nqo1 by p62-mediated Nrf2 activation facilitates p53-dependent mitotic catastrophe. Biochem Biophys Res Commun 412:347352 .

Chakraborty C, Doss CGP, Chen L and Zhu H (2014) Evaluating protein-protein interaction (PPI) networks for diseases pathway, target discovery, and drug-design using 'in silico pharmacology'. Curr Protein Pept Sci 15:561-571.

Chomczynski P and Sacchi N (2006) The single-step method of RNA isolation by acid guanidinium thiocyanate-phenolchloroform extraction: Twenty-something years on. Nat Protoc 1:581.

Copple IM, Lister A, Obeng AD, Kitteringham NR, Jenkins RE, Layfield R, Foster BJ, Goldring CE and Park BK (2010) Physical and functional interaction of sequestosome 1 with Keap1 regulates the Keap1-Nrf2 cell defense pathway. J Biol Chem 285:16782-16788.

Dinkova-Kostova AT and Talalay P (2010) NAD(P)H:quinone acceptor oxidoreductase 1 (NQO1), a multifunctional antioxidant enzyme and exceptionally versatile cytoprotector. Arch Biochem Biophys 501:116-123.

Dovzhik AD, Zolotukhin PV, Mayboroda EA, Mashkina EV and Aleksandrova AA (2014) Assessing the regulation dependencies of thioredoxin 1 transcript variants by means of interactomic dynamic-with-induction profiling approach. Zhiviye i biokostnye systemy (Living and life-reconditioned systems) 20146:8.

Erol Ö, Arda N and Erdem G (2012) Phenols of virgin olive oil protects nuclear DNA against oxidative damage in HeLa cells. Food Chem Toxicol 50:3475-3479.

Jain A, Lamark T, Sjøttem E, Larsen KB, Awuh JA, Øvervatn A, McMahon M, Hayes JD and Johansen T (2010) p62/SQSTM1 is a target gene for transcription factor NRF2 and creates a positive feedback loop by inducing antioxidant response element-driven gene transcription. J Biol Chem 285:22576-22591.

Jaiswal AK (2000) Regulation of genes encoding NAD(P)H: Quinone oxidoreductases. Free Radic Biol Med 29:254-262.

Jyrkkänen HK, Kuosmanen S, Heinäniemi M, Laitinen H, Kansanen E, Mella-Aho E, Leinonen H, Ylä-Herttuala S and Levonen AL (2011) Novel insights into the regulation of an- tioxidant-response-element-mediated gene expression by electrophiles: Induction of the transcriptional repressor BACH1 by Nrf2. Biochem J 440:167-174.

Kim HJ, Zheng M, Kim SK, Cho JJ, Shin CH, Joe Y and Chung HT (2011) CO/HO-1 Induces NQO-1 expression via Nrf2 activation. Immune Netw 11:376-382.

Kurinna S and Werner S (2015) NRF2 and microRNAs: New but awaited relations. Biochem Soc Trans 43:595-601.

Laloo B, Maurel M, Jalvy-Delvaille S, Sagliocco F and Grosset CF (2010) Analysis of post-transcriptional regulation using the FunREG method. Biochem Soc Trans 38:1608-1614.

Lerdrup M, Holmberg C, Dietrich N, Shaulian E, Herdegen T, Jäättelä M and Kallunki T (2005) Depletion of the AP-1 repressor JDP2 induces cell death similar to apoptosis. Biochim Biophys Acta 1745:29-37.

Lin YH and Bundschuh R (2013) Interplay between singlestranded binding proteins on RNA secondary structure. Phys Rev E Stat Nonlin Soft Matter Phys 88:052707.

Liu Y, Timani K, Ou X, Broxmeyer HE and He JJ (2013) C-MYC controlled TIP110 protein expression regulates OCT4 mRNA splicing in human embryonic stem cells. Stem Cells Dev 22:689-694.

Luco RF, Pan Q, Tominaga K, Blencowe BJ, Pereira-Smith OM and Misteli T (2010) Regulation of alternative splicing by histone modifications. Science 327:996-1000.

MacLeod AK, McMahon M, Plummer SM, Higgins LG, Penning TM, Igarashi K and Hayes JD (2009) Characterization of the cancer chemopreventive NRF2-dependent gene battery in human keratinocytes: Demonstration that the KEAP1-NRF2 pathway, and not the BACH1-NRF2 pathway, controls cytoprotection against electrophiles as well as redox-cycling compounds. Carcinogenesis 30:1571-1580.

Malakooti J, Dahdal RY, Dudeja PK, Layden TJ and Ramaswamy $\mathrm{K}$ (2001) The human $\mathrm{Na}(+) / \mathrm{H}(+)$ exchanger NHE2 gene: Genomic organization and promoter characterization. Am J Physiol Gastrointest Liver Physiol 280:G763-G773.

Marrot L, Jones C, Perez P and Meunier JR (2008) The significance of Nrf2 pathway in (photo)-oxidative stress response in melanocytes and keratinocytes of the human epidermis. Pigment Cell Melanoma Res 21:79-88.

Ozretić P, Bisio A, Musani V, Trnski D, Sabol M, Levanat S and Inga A (2015) Regulation of human PTCH1b expression by different 5' untranslated region cis-regulatory elements. RNA Biol 12:290-304.

Pan J, Jin C, Murata T and Yokoyama KK (2003) Sequence specific transcription factor, JDP2 interacts with histone and inhibits p300-mediated histone acetylation. Nucleic Acids Res 2003(Suppl 3):305-306.

Rattenbacher B and Bohjanen PR (2012) Evaluating posttranscriptional regulation of cytokine genes. Methods Mol Biol 820:71-89.

Sena JA, Wang L, Heasley LE and Hu CJ (2014) Hypoxia regulates alternative splicing of HIF and non-HIF target genes. Mol Cancer Res 12:1233-1243.

Shim J and Karin M (2002) The control of mRNA stability in response to extracellular stimuli. Mol Cells 14:323-331.

Shimizu H, Horimoto Y, Arakawa A, Sonoue H, Kurata M, Kosaka T, Nakai K, Himuro T, Tokuda E, Takahashi Y, et al. (2015) Application of a 70-gene expression profile to Japanese breast cancer patients. Breast Care 10:118-122. 
Shkurat T, Zolotukhin P, Lebedeva U, Kuzminova O, Belanova A, Korinfskaya S, Chmykhalo V and Aleksandrova A (2014) Prospects of applied interactomics of oxidative status for molecular pathophysiology and pharmacology. Proceedings of EMBO Conference From Functional Genomics to Systems Biology, Heidelberg, p. 249.

Shukla SJ, Huang R, Simmons SO, Tice RR, Witt KL, Vanleer D, Ramabhadran R, Austin CP and Xia M (2012) Profiling environmental chemicals for activity in the antioxidant response element signaling pathway using a high throughput screening approach. Environ Health Perspect 120:11501156.

Sibhatu MB, Smitherman PK, Townsend AJ and Morrow CS (2008) Expression of MRP1 and GSTP1-1 modulate the acute cellular response to treatment with the chemopreventive isothiocyanate, sulforaphane. Carcinogenesis 29:807-815.

Siegel D, Gustafson DL, Dehn DL, Han JY, Boonchoong P, Berliner LJ and Ross D (2004) NAD(P)H:quinone oxidoreductase 1: Role as a superoxide scavenger. Mol Pharmacol 65:1238-1247.

Siegel D, Kepa JK and Ross D (2012) NAD(P)H:quinone oxidoreductase 1 (NQO1) localizes to the mitotic spindle in human cells. PLoS One 7:e44861.

Singh A, Rangasamy T, Thimmulappa RK, Lee H, Osburn WO, Brigelius-Flohé R, Kensler TW, Yamamoto M and Biswal S (2006) Glutathione peroxidase 2, the major cigarette smoke-inducible isoform of GPX in lungs, is regulated by Nrf2. Am J Respir Cell Mol Biol 35:639-650.

Subramaniam SR and Ellis EM (2011) Esculetin-induced protection of human hepatoma HepG2 cells against hydrogen peroxide is associated with the Nrf2-dependent induction of the NAD(P)H: Quinone oxidoreductase 1 gene. Toxicol Appl Pharmacol 250:130-136.

Sykiotis GP and Bohmann D (2010) Stress-activated cap'n'collar transcription factors in aging and human disease. Sci Signal 3:re3.

Tanigawa $\mathrm{S}$, Lee $\mathrm{CH}$, Lin $\mathrm{CS}, \mathrm{Ku} \mathrm{CC}$, Hasegawa $\mathrm{H}$, Qin $\mathrm{S}$, Kawahara A, Korenori Y, Miyamori K, Noguchi M, et al. (2013) Jun dimerization protein 2 is a critical component of the Nrf2/MafK complex regulating the response to ROS homeostasis. Cell Death Dis 4:e921.

Trombetta-Lima M, Winnischofer SM, Demasi MA, Astorino Filho R, Carreira AC, Wei B, Assis-Ribas TD, Konig MS, Bowman-Colin C, Oba-Shinjo SM et al. (2015) Isolation and characterization of novel RECK tumor suppressor gene splice variants. Oncotarget 6:33120-133.

Venugopal R and Jaiswal AK (1996) Nrf1 and Nrf2 positively and c-Fos and Fra1 negatively regulate the human antioxidant response element-mediated expression of
NAD(P)H:quinone oxidoreductase1 gene. Proc Natl Acad Sci U S A 93:14960-14965.

Wang S, Zheng W, Liu X, Xue P, Jiang S, Lu D, Zhang Q, He G, Pi J, Andersen ME, et al. (2014) Lodoacetic acid activates Nrf2-mediated antioxidant response in vitro and in vivo. Environ Sci Technol 48:13478-13488.

Winski SL, Koutalos Y, Bentley DL and Ross D (2002) Subcellular localization of $\mathrm{NAD}(\mathrm{P}) \mathrm{H}$ :quinone oxidoreductase 1 in human cancer cells. Cancer Res 62:1420-1424.

Wu RP, Hayashi T, Cottam HB, Jin G, Yao S, Wu CC, Rosenbach MD, Corr M, Schwab RB and Carson DA (2010) Nrf2 responses and the therapeutic selectivity of electrophilic compounds in chronic lymphocytic leukemia. Proc Natl Acad Sci U S A 107:7479-7484.

Yadav AK, Vashishta V, Joshi N and Taneja P (2014) AR-A 014418 Used against GSK3beta downregulates expression of hnRNPA1 and SF2/ASF splicing factors. J Oncol 2014:695325.

Yao K, Goldschmidt R, Turk M, Wesseling J, Stork-Sloots L, de Snoo F and Cristofanilli M (2015) Molecular subtyping improves diagnostic stratification of patients with primary breast cancer into prognostically defined risk groups. Breast Cancer Res Treat 154:81-88.

Zhang Q, Pi J, Woods CG and Andersen ME (2010) A systems biology perspective on Nrf2-mediated antioxidant response. Toxicol Appl Pharmacol 244:84-97.

Zolotukhin P, Aleksandrova A, Goncharova A, Shestopalov A, Rymashevskiy A and Shkurat T (2014a) Oxidative status shifts in uterine cervical incompetence patients. Syst Biol Reprod Med 60:98-104.

Zolotukhin PV, Dovzhik AD, Lebedeva UA, Kuzminova ON, Mashkina EV, Aleksandrova AA and Shkurat TP (2014b) Testing the concept of the interatomic status of the NFE2L2/AP1 pathway as a systemic biomarker for examination stress. Mol Diagn Ther 18:355-369.

\section{Internet Resources}

NCBI Gene Database, http://ncbi.nlm.nih.gov/gene (December 2015).

RNA fold web instrument, http://rna.tbi.univie.ac.at/cgi-bin/RNAfold.cgi (May 2015).

\section{Supplementary Material}

The following online material is available for this article: Figure S1 - RNA integrity testing results.

Table S1 - Primer sequences used in the study.

Associate Editor: Carlos R. Machado

License information: This is an open-access article distributed under the terms of the Creative Commons Attribution License (type CC-BY), which permits unrestricted use, distribution and reproduction in any medium, provided the original article is properly cited. 